\title{
Sites Internet des Archives nationales du Canada et des Archives nationales du Québec < http://www.archives.ca> et <http://www.anq.gouv.qc.ca>. Sites évalués à la fin mars 2003.
}

\section{Donald Fyson}

Volume 56, numéro 4, printemps 2003

URI : https://id.erudit.org/iderudit/007801ar

DOI : https://doi.org/10.7202/007801ar

Aller au sommaire du numéro

Éditeur(s)

Institut d'histoire de l'Amérique française

ISSN

0035-2357 (imprimé)

1492-1383 (numérique)

Découvrir la revue

Citer ce compte rendu

Fyson, D. (2003). Compte rendu de [Sites Internet des Archives nationales du Canada et des Archives nationales du Québec <http://www.archives.ca> et $<$ http://www.anq.gouv.qc.ca>. Sites évalués à la fin mars 2003.] Revue d'histoire de l'Amérique française, 56(4), 577-579. https://doi.org/10.7202/007801ar 


\section{COM P T E R E N D U S DE SITES I N T E R N ET}

Sites Internet des Archives nationales du Canada et des Archives nationales du Québec <http://www.archives.ca> et <http://www.anq.gouv.qc.ca>. Sites évalués à la fin mars 2003.

Les sites Internet respectifs des Archives nationales du Canada (ANC) et des Archives nationales du Québec (ANQ) remplissent plusieurs fonctions : entre autres, la diffusion de l'information sur les organismes, la pédagogie et la vulgarisation et les services aux chercheurs. C'est sur cette dernière fonction que porte ce compte rendu. Le chercheur trouve d'abord, au cœur des deux sites, les instruments de recherche permettant de repérer les documents. Aux ANC, il s'agit de la vingtaine de banques de données regroupées sous la rubrique ArchiviaNet, y compris l'inventaire général, des instruments de recherche généraux comme ceux sur les photographies ou les archives coloniales et certaines banques de données plus spécialisées. Chaque banque a sa propre interface de recherche particulière, ce qui peut porter à confusion, mais les interfaces sont assez simples (généralement, une recherche par mots clés dans l'ensemble de la notice, y compris la description) et sont accompagnées de fonctions d'aide détaillées. On notera tout de même quelques limites : entre autres, la plupart des instruments de recherche plus généraux ne permettent pas une recherche ou un tri des résultats par date et il est difficile de retrouver des fonds dans l'inventaire général si on n'a que les anciennes cotes RG et MG. Soulignons aussi l'absence de certains instruments essentiels, comme un catalogue détaillé des microfilms (à part ceux des recensements), qui permettra de commander des bobines précises sans une recherche sur place. Dans l'ensemble, toutefois, il s'agit d'un outil de recherche satisfaisant.

On ne peut en dire autant du site des ANQ. L'équivalent de l'inventaire général, Pistard Web, a tout pour décourager l'utilisateur. D’abord, il y a la 
lenteur de la banque, qui semble hébergée sur un serveur Oracle commercial plutôt que sur un serveur dédié : quand le système n'est pas entièrement inaccessible, ce qui est souvent le cas, les recherches peuvent prendre plusieurs minutes avant d'aboutir. Dans mes démonstrations pédagogiques, j'ai pris l'habitude de lancer une recherche Pistard Web dans une fenêtre à part, passer à autre chose et revenir plus tard pour vérifier si le système a répondu à la requête. Comme aux $\mathrm{ANC}$, l'interface de recherche est simple mais elle comporte des limites importantes : aucune recherche par titre du fonds ou par cote, aucune recherche dans le champ de description (une limite de Pistard lui-même), etc. Notons toutefois que Pistard Web permet de limiter la recherche par date. De même, les résultats de la recherche sont loin d'être conviviaux : il faut d'abord passer par une liste de sujets avant d'accéder aux descriptions des fonds, et qu'importe la logique interne de la chose, la rubrique «sujets refusés» ne peut que rebuter l'utilisateur. En fin de compte, il est dommage qu'il soit plus efficace de faire une recherche pour les collections des ANQ dans le meta-répertoire du RDAQ (<http:/ / www.rdaq.qc.ca/ >) qui contient l'essentiel des notices de Pistard et qui permet aussi une recherche dans la partie descriptive de la notice (mais qui, curieusement, ne donne pas les dates extrêmes des fonds provenant de Pistard, ce qui en limite l'utilité). À part Pistard Web, le site des ANQ présente deux ou trois autres instruments de recherche, utiles en soi mais à portée limitée.

Au-delà de l'identification des documents pertinents à une recherche, les sites Internet des centres d'archives peuvent aussi permettre un accès direct au contenu, sous forme d'images ou de transcriptions. Au Québec et au Canada, l'accent a été mis pendant longtemps sur la numérisation des documents publiés, de sorte que la disponibilité sur Internet des sources inédites est encore limitée. Toutefois, aux ANC, ce volet est en expansion constante depuis plusieurs années, en commençant par les œuvres d'art et les photographies, dont plusieurs milliers sont disponibles, auxquelles s'ajoutent maintenant plusieurs fonds ou séries numérisés au complet, allant des recensements de 1901 et 1906, en passant par les journaux intimes de Mackenzie King et les documents des cabinets fédéraux, jusqu’à des fonds spécialisés comme celui de la seigneurie de Sorel. Ces documents numérisés sont accessibles directement à partir des instruments de recherche associés, ce qui rend très facile leur consultation. La sélection des documents numérisés est aussi assez bien équilibrée, ce qui donne une collection utile à la fois pour les généalogistes et pour les historiens (bien qu'on notera un certain accent sur les sources d'intérêt généalogique), tant 
pour la pédagogie que pour la recherche. Sur le site des ANQ, par contre, la collection numérisée est composée essentiellement de la petite sélection d'images et de documents qui composent les expositions virtuelles; collection intéressante mais très limitée.

Dans l'ensemble, on comprend assez mal le retard du site des ANQ. Bien que les ANC disposent d'un budget beaucoup plus substantiel, même les sites de plusieurs archives provinciales sont mieux garnis que celui des ANQ, notamment ceux de l'Ontario et de la Colombie-Britannique. Qu'importe les limites du système Pistard lui-même, il y a sans doute moyen d'améliorer son interface Web. Quant au contenu numérique, ce n'est pas la matière brute qui manque. Les ANQ ont créé de nombreux instruments de recherche spécialisés, dont certains sous forme de banques de données (notamment les diverses banques créées en partenariat avec le secteur privé et les banques ÉDIBASE et MÉDIABASE) et sans doute un grand nombre d'autres dans des formats électroniques plus simples, comme des fichiers de traitement de texte. Les collections des ANQ ont aussi fait l'objet de plusieurs projets de reproduction électronique, allant de la numérisation des Rapports de l'archiviste aux 100000 photographies de MÉDIABASE sur vidéodisque. Mais c'est la diffusion plus restreinte qui a été privilégiée, notamment le cédérom, la banque de données locale ou encore (dans le cas des instruments de recherche) la microfiche. Sans doute est-ce en partie une question de commercialisation : on comprend bien la réticence de partenaires privés de rendre gratuitement disponible ce qui est en partie leur produit. Les difficultés techniques ne sont pas non plus à ignorer : la numérisation d'images sur vidéodisque analogique, par exemple, semble complexe, bien que possible. Mais pour le chercheur, le résultat est tout de même décevant; les quelques exceptions, comme l'inventaire du fonds Maurice Proulx ou l'excellent Guide des Archives judiciaires, nous laissent sur notre faim.

En somme, le site des ANC est bien garni, toujours en développement et, malgré quelques inconvénients, d'une fonctionnalité satisfaisante. Le site actuel des ANQ a relativement peu de contenu et certaines de ses composantes ne desservent pas bien le chercheur; malheureusement, le site ne valorise que très peu la richesse des collections des ANQ et l'excellence de son travail archivistique. Le vent semble commencer à tourner : le site a actuellement un sondage qui demande aux utilisateurs des ANQ quels projets de numérisation et de mise en ligne devraient être privilégiés. Souhaitons que cela se concrétise rapidement.

DONALD FYSON

Département d'histoire Université Laval 